\title{
Triorchidism: which therapy?
}

\author{
Eugenia Piro, Laura Abati, Veronica Zocca, Marta Brugnoni, Antonio D’Alessio \\ Department of Pediatric Surgery, Local Health Unit, Legnano (MI), Italy
}

\begin{abstract}
Polyorchidism is an anomaly characterized by more than two gonads; triorchidism is the most common variant. Its management is controversial, mostly when surgical treatment is occasional. CB, 14 year-old, came to the hospital due to right-sided testicular torsion. During surgery, testis was rotated and the contralateral testis, which presented as an anatomically continuum with a gonadic structure similar to the other testes but with a smaller diameter, was fixed. We performed biopsy on both left testes and decided to preserve the supernumerary one. Following the anatomic and functional classification of polyorchidism by Singer, preservation is justified on the grounds of the presence of a supernumerary testis that drains into the epididymis of the normal testis, merging into one single deferent duct (Singer Type 1). At biopsy, both testes had a valid spermatogenic asset. The diagnostic follow-up at 6 and 12 months did not show any pathological alteration. Diagnosis of polyorchidism is occasional. Its treatment varies depending on the site, dimension, and anatomy of the drainage system of the supernumerary testis. If the supernumerary testis is preserved, a standardized diagnostic follow-up is recommended.
\end{abstract}

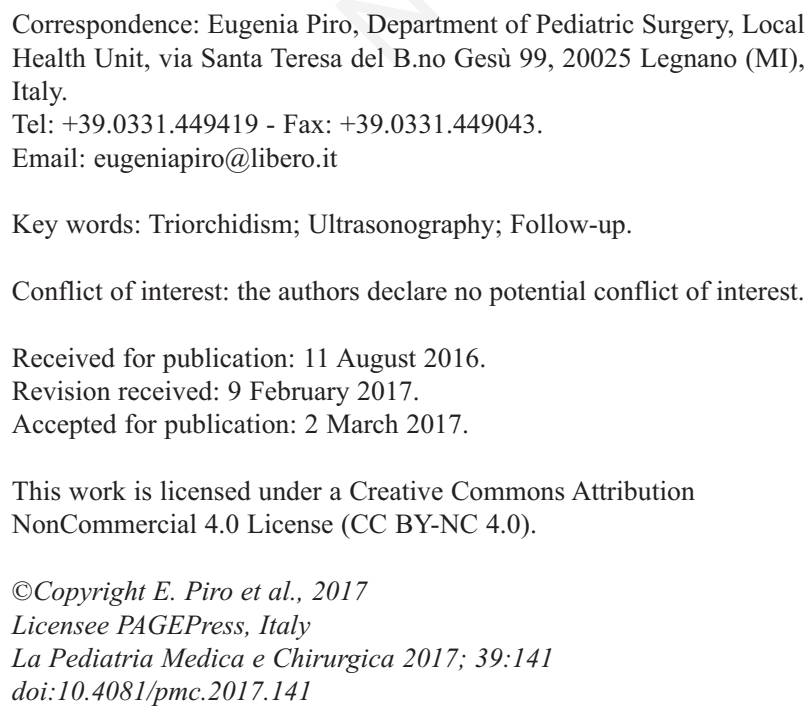

\section{Introduzione}

Il poliorchidismo, condizione caratterizzata dalla presenza di più di due gonadi, è una rara anomalia congenita del sistema urogenitale. Il triorchidismo ne è la presentazione più comune ${ }^{1}$ anche se sono stati descritti pazienti portatori fino a 5 gonadi. ${ }^{2}$ In letteratura ne vengono riportati meno di 200 casi. ${ }^{3}$ Il riscontro di gonade sovranumeraria, che solo in alcuni può appalesarsi come massa dolente, a sede scrotale o inguinale, nella maggior parte dei pazienti è asintomatico, reperto occasionale, in corso di trattamento chirurgico della patologia associata, situazione che ne rende controverso il suo management. La patologia più frequentemente associata al poliorchidismo è rappresentata da criptorchidismo $(40 \%)$, ernia inguinale $(30 \%)$, torsione testicolare $(13 \%)$ ed idrocele $(9 \%)$, seguite da ipospadia $(<1 \%)$, varicocele $(<1 \%)$, neoplasie $(<1 \%){ }^{4}$

\section{Caso Clinico}

Un ragazzo di 14 anni di origine moldava giungeva alla nostra osservazione per insorgenza improvvisa di algia a livello del testicolo destro associata a vomito. Non veniva riferita disuria. All'esame clinico l'emiscroto di destra appariva edematoso e la gonade omolaterale, dolente, si presentava altoposta rispetto alla controlaterale e disposta a batacchio di campana. La palpazione della gonade controlaterale permetteva di apprezzare una tumefazione di circa $20 \mathrm{~mm}$ x $15 \mathrm{~mm}$, di consistenza elastica, non dolente, apparentemente a carico dell'epididimo e descritta come verosimile spermatocele sinistro. Si eseguiva manovra di derotazione manuale della gonade destra ma con parziale risoluzione della torsione, quindi si decideva prontamente di sottoporre il paziente ad esplorazione chirurgica dell'emiscroto di destra, soprassedendo alla valutazione ecografica. Il quadro clinico, infatti, non dava adito a dubbi diagnostici in merito alla torsione gonadica destra, tanto da dover procrastinare l'intervento, con aumento del rischio di perdita della gonade, per eseguire un'indagine che non avrebbe comunque modificato l'atteggiamento chirurgico adottato. Questo confermava la torsione funicolare che quindi veniva definitivamente risolta, con risoluzione della stasi venosa di cui risultava preda la gonade, per il resto indenne da altre alterazioni macroscopiche e con diametri nella norma. Si pro- 
cedeva quindi alla fissazione del testicolo sinistro. Quest'ultimo, con diametri pari a quelli del controlaterale, però si presentava in continuità anatomica con analoga struttura gonadica, posta in posizione prossimale, di diametro inferiore, drenante nell'epididimo della gonade sinistra normale.

Si eseguiva biopsia su entrambi i testicoli di sinistra decidendo di conservare la gonade soprannumeraria e fissandole entrambe (Figura 1).

L'esame istologico dei due parenchimi testicolari risultò sovrapponibile, presentando entrambi tubuli seminiferi con cellule seminifere in tutte le fasi di maturazione (spermatogoni, spermatociti, spermatozoi) e cellule del Sertoli con regolare morfologia e struttura.

L'ecografia eseguita a distanza di 3 e 6 mesi non ha evidenziato alcuna alterazione del parenchima della gonade sinistra sovranumeraraia.

\section{Discussione}

Il primo caso di poliorchidismo, confermato come tale istologicamente, è quello descritto da Lane nel 1895, anche se già nel 1670 Blasius descriveva il riscontro di una gonade sovranumeraria in corso di un'autopsia di routine. L'età in cui tale condizione può riscontrarsi è compresa tra un vasto range che va dai 3 ai 75 anni, ma nel 50\% dei casi il riscontro avviene tra i 15 e i 25 anni mentre sono rari i casi d'età pari o inferiore ai 2 anni.5,6

La maggior parte delle gonadi soprannumerarie sono localizzate in regione scrotale $(66 \%)$, seguite da quelle a sede inguinale $(23 \%)$ e quelle a sede addominale $(9 \%)^{7}$
La più comune presentazione del poliorchidismo è il triorchidismo con gonade sovranumeraria generalmente localizzata a livello del lato sinistro $(>75 \%)^{8}$ e avente diametro spesso più piccolo di entrambe le altre gonadi, sia l'omolaterale che la controlaterale. ${ }^{9}$ Descritto un unico caso di poliorchidismo con tre gonadi omolaterali a localizzazione destra e anorchia controlaterale. ${ }^{10}$

L'eziologia di questa rara malformazione urogenitale non è chiara e le già poche teorie proposte in merito non spiegano in maniera univoca l'eterogeneità delle varianti anatomiche descritte. In un embrione normale intorno alla sesta settimana di vita le gonadi primordiali si sviluppano partendo dalla cresta genitale migrando verso i dotti mesonefritici. All'ottava settimana, la determinazione genetica in senso maschile permette la persistenza di questi ultimi da cui avranno origine l'epididimo e il deferente. L'esatto meccanismo per cui origina una condizione di poliorchidismo non è noto ma verosimilmente è da imputarsi a un'anomala divisione, longitudinale o trasversa della cresta genitale per lo sviluppo di bande peritoneali. $5,6,9$

Il primo a formulare una classificazione anatomica per il poliorchidismo è stato Leung, basandosi e relazionandone i vari tipi alle variazioni embriologiche ipotizzate. Questi ne descrive 4 tipi. Il tipo I è identificabile con quella condizione in cui la gonade sovranumeraria non è provvista né di epididimo né di dotto deferente, non ha comunicazioni con la gonade normale ed è embriologicamente il risultato di una divisione delle cellule della cresta genitale senza una loro migrazione verso il dotto mesonefritico; nel tipo II la gonade sovranumeraria drena nell'epidididmo della gonade normale che si continua con il suo dotto deferente; tale condizione si verificherebbe quando la divisione a carico della cresta genitale avviene lì dove la gonade primordiale entra
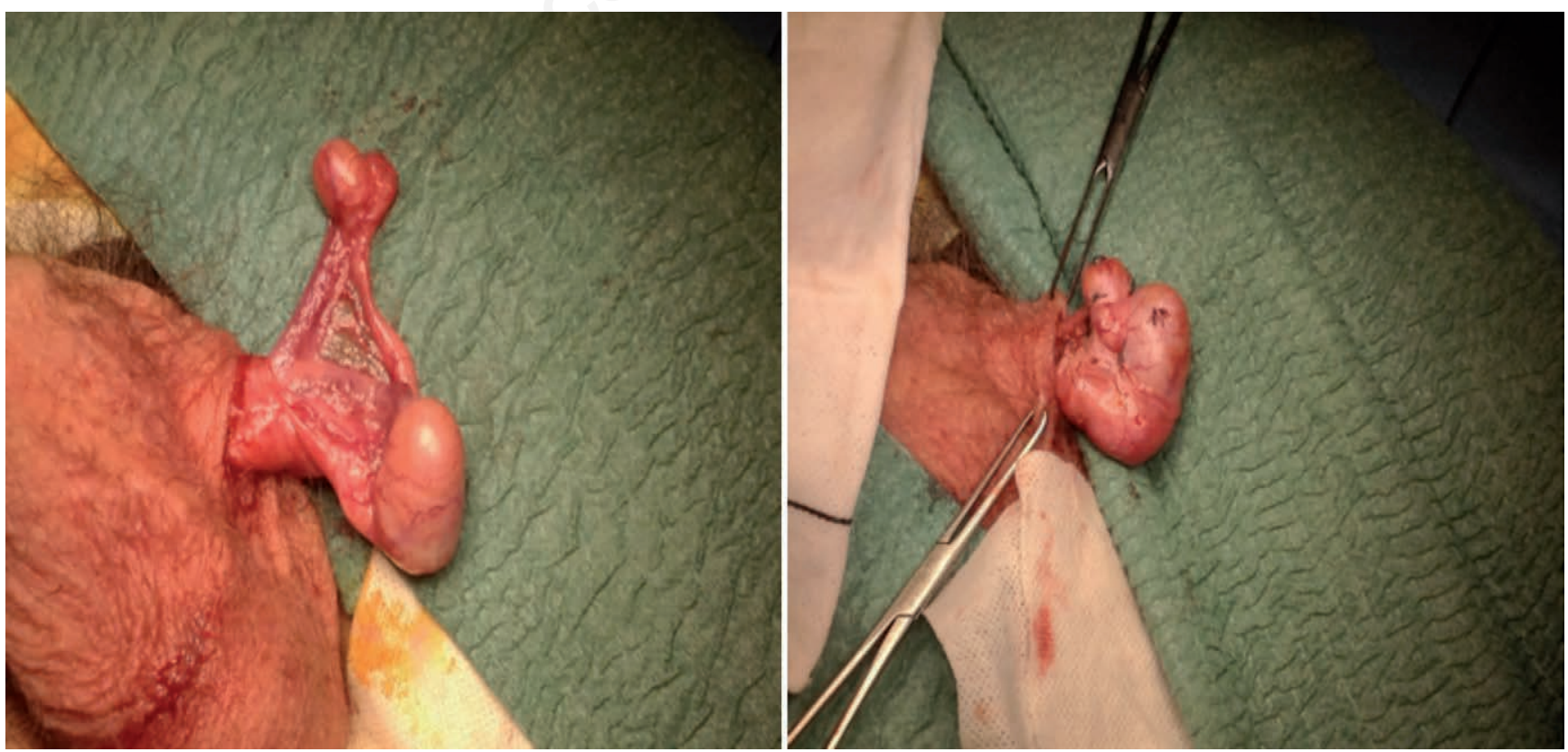

Figura 1. Biopsia su entrambi i testicoli di sinistra. 
in contatto con il dotto mesonefritico; il tipo III vede la gonade sovranumeraria provvista di un suo epididimo che drena, insieme all'epididimo della gonade normale, nel dotto deferente di questa; questo tipo è la conseguenza embriologica di una completa divisione trasversa della cresta genitale e del dotto mesonefritico; il tipo IV, legato ad una divisione longitudinale competa sia della cresta genitale che del dotto mesonefritico, si identifica con una duplicazione completa di didimo, epididimo e dotto deferente. ${ }^{11}$

Singer et al. proposero più schematicamente una classificazione in cui le caratteristiche anatomiche della gonade sovranumeraraia ne giustificavano le varianti funzionali, capaci in quanto tali di dettare o quanto meno modificare il management della patologia. La classificazione proposta da Singer per il poliorchidismo ne distingue due tipi. Un tipo I, quando la gonade sovranumeraria, in relazione con la gonade normale nel cui epididimo e/o dotto deferente drena, conserva un potenziale riproduttivo; tipo II, una gonade sovranumeraria priva di alcun sistema di drenaggio e relazione con la gonade normale e quindi senza alcun potenziale riproduttivo. ${ }^{12}$

Il riscontro, spesso accidentale, in genere in corso di altra patologia (ernia inguinale, idrocele, criptorchidismo), di una condizione di poliorchidismo, ne rende controverso il management. La gonade sovranumeraria si pone in diagnosi differenziale con altre masse scrotali extratesticolari quali spermatocele, idrocele, adenoma, cistoadenoma papillare. L'ecografia a riguardo è dirimente. Nel caso dell'associazione con la stessa torsione, che è una condizione acuta, l'esecuzione dell'ecografia non deve ritardare il trattamento della stessa, pena l'aumentato rischio di perdita del testicolo. ${ }^{13}$ In presenza di un reperto diagnostico occasionale, senza altra necessità di dover intervenire, adottare un atteggiamento conservativo sembra attualmente la scelta più giusta. In considerazione dei dati riportati in letteratura e in base ai quali i quadri di poliorchidismo sono associati a un accertato aumento di infertilità $(20 \%)$, valutare la potenzialità di fertilità della gonade sovranumeraria (tipo I di Singer), giustifica mantenere in situ la gonade soprannumeraria e monitorarla con risonanza magnetica $(\mathrm{RMN})$ per tenere sotto controllo il pur non sottovalutabile rischio di malignità che in questi casi è compreso tra il 4 e il 7\%, a fronte di quello presente nella popolazione generale, pari a $0,004 \%$, e a quello dei criptorchidi, che è dello $0,045 \% .5,6 \mathrm{Il}$ riscontro di neoplasia maligna in gonadi sovranumerarie è maggiore se queste hanno sede extrascrotale (addominale o inguinale), con presentazione di comparsa anticipata a 19 anni di età a fronte dei 34 anni per i tumori a cellule germinali dei testicoli normali per numero e sede. ${ }^{14}$ Le neoplasie riscontrate sono rappresentate da seminoma, corioncarcinoma e teratoma. ${ }^{9}$ Vale ugualmente non ricorrere all'exeresi della gonade sovranumeraria, quando, in corso di intervento per altra patologia, la gonade si presenta vitale e, come sopra, ascrivibile ad un tipo I di Singer, fissandola, invece, perché è descritta un'aumentata incidenza di torsione a carico delle gonadi sovranumerarie. È giustificato ricorrere alla sua rimozione chirurgica se è classificabile come un tipo II di Singer e quando è criptorchide o ectopica.
Il follow up proposto per questi pazienti prevede l'esecuzione di un'ecografia e una valutazione clinica con adeguata palpazione una volta al mese per i primi 3 mesi, poi ogni 6 mesi per i successivi 2 anni e dopo l'adolescenza ogni 2 anni. Al compimento del diciottesimo anno di età all'esame clinico e alla valutazione ecografia si aggiungeranno l'esame del liquido seminale, e dei livelli sierici di testosterone e dell'ormone follicolo stimolante, a seguire, un'ecografia ogni 2 anni.

\section{Conclusioni}

La diagnosi di poliorchidismo è spesso occasionale. Nella scelta del suo management entrano in gioco diversi fattori: il sistema di drenaggio, quindi le potenzialità fertili della gonade sovranumeraria, e la sua sede. In accordo con quanto evidenziato in letteratura non si ritiene opportuno ricorrere all'intervento chirurgico, se non per eventuale patologia concomitante. In particolare nei casi di gonade sovranumeraria criptorchide, dove l'incidenza di neoplasia maligna è maggiore. Nei casi di poliorchidismo non complicato, l'adozione di un trattamento conservativo, dove la valutazione ecografica e/o il ricorso alla RMN ne consentono un'osservazione scevra da rischi, appare la scelta più razionale.

\section{Bibliografia}

1. Athwal S, Tailor J, Lakhoo K. Triorchidism at orchidopexy: a case report. J Med Case Report 2008;2:247.

2. Kumar K, Das D, Shivaraj. Triorchidism with torsion. Ann Med Health Sci Res 2012;2:199-201.

3. Abduljabbar AH. A case report: triorchidism; is a rare mistaken cause for extra testicular neoplasm. Urology Case Rep 2015;3:89-1.

4. Sharma AK, Mathur P. Polyorchidism. Pediatr Surg Int 1994;9:531.

5. Kheirandish P, Chinegwundoh F. An unusual case of triorchidism. JRSM Short Rep 2010;1:55.

6. Tonape T, Singh G, Koushik P, Tumepalli T. Triorchidism: a rare genitourinari abnormality. J Surg Tech Case Rep 2012;4:126-8.

7. Bergholz R, Wenke K. Polyorchidism: a meta-analysis. J Urol 2009;182:2422-7.

8. Thyoka M, Lall A, Godse A. Polyorchidism: a torted right-sided supernumerary testis. Brit Med J Case Rep 2013;2013:008600.

9. Tatli D, Numanoglu KV. Polyorchidism presenting with inguinal hernia ad hypospadias. Case Rep Urol 2013; 2013:917050.

10. Vijayanadh O, Shanbhogue AK, Doherty GP. An unusual case of polyorchidism with three homolateral testes and controlateral anorchia. Eur J Radiol Extra 2009; 72: 129-31. 
11. Leung AK. Polyorchidism. Am Fam Physician 1988;38: 153-6.

12. Singer BR, Donaldson JG, Jackson DS. Polyorchidism: functional classification and management strategy. Urology 1992;39:384-8.
13. Ayvaz OD, Clayir AC, Moralioglu S, et al. Four-year retrospective look for acute scrotal pathologies. North Clin Istanbul 2015;2:182-8.

14. Bergholz R, Wenke K. Polyorchidism: a meta-analysis. Pediatr Urol 2009;182:2422-7. 